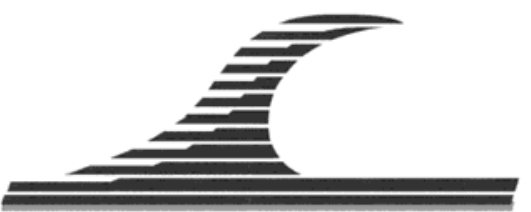

\title{
The method of decomposition domain: application to the racking problem
}

\author{
Soumia MORDANE ${ }^{1}$, Khalid ADNAOUI ${ }^{2}$, Mohamed LOUKILI ${ }^{1}$, \\ Noureddine TOUNSI ${ }^{2}$, Mohamed CHAGDALI ${ }^{1}$
}

1. LPPPC, Faculté des Sciences Ben M’Sik, Casablanca, Maroc. mordanesoumia@yahoo.fr

2. MITI, Faculté des Sciences Ben M’Sik, Casablanca, Maroc.

\begin{abstract}
:
In this work, we present The method of decomposition domain to solve the NavierStokes equations. Two applications are presented to illustrate the advantages of the method: the first concerns the jet emitted from the bottom in an open channel and the second deals with the lateral racking problem. These two applications concern the problem of creating artificial circulation of the water masses in order to homogenize the physical parameters of the medium.

In applying the decomposition method, the field of studies is broken down into sub areas to take better account of the physical processes and the numerical methods suited to their applications. Two numerical methods are used, a finite difference near the wall and a particle method in high traffic areas. In the interfaces of sub-domains, using coupling conditions by the particle-mesh method.
\end{abstract}

Translated version of a paper in French (DOI reference: http://dx.doi.org/10.5150/cmcm.2015.063), presented during the edition 3 of the Coastal and Maritime Mediterranean Conference,

(25-27 November 2015) in Ferrara, Italy.

Received 28 July 2015, accepted 11 December 2015, available online 18 July 2016. 


\section{Introduction}

The direct resolution of Navier-Stokes equations by the finite difference method, poses a large challenge for high Reynolds numbers. Other methods are better suited to treat the flows of viscous fluids in the case of high recirculation speed. Among these methods, particle methods are cited (HUBERSON \& CHOQUIN, 1988). The combination of these two methods can improve the resolution of the Navier-Stokes equations especially for large areas of study. This work uses the decomposition method of the area for two applications related to the media stratification problem in a basin (MANGOUB, 2007; ADNAOUI et al., 2014). The use of a jet from the bottom and the side racking.

\section{Domain Decomposition Method}

It seeks to solve the following Navier-Stokes equations:

$$
\begin{aligned}
& \frac{\partial \omega}{\partial t}+(\vec{V} \cdot \vec{\nabla}) \omega=\frac{1}{\mathfrak{R}_{e}} \Delta \omega+\frac{1}{F r^{2}}\left[\vec{\nabla} C \wedge \frac{\vec{g}}{\|\vec{g}\|}\right] \cdot \vec{k} \\
& \Delta \psi=-\omega \\
& \vec{V}=\vec{\nabla} \wedge(-\psi \vec{k}) \\
& \frac{\partial C}{\partial t}+(\vec{V} \cdot \vec{\nabla}) C=\frac{1}{\mathfrak{R} e S c} \Delta C
\end{aligned}
$$

in which $\vec{\nabla}$ et $\Delta$ are respectively the gradient operator and the Laplacian operator, the vector $\vec{k}$ is directly perpendicular to the flow map, $\vec{U}$ is the velocity vector, $\psi$ is the current function, $\omega$ is the vorticity function such that $\vec{\omega}=\omega \vec{k}=\vec{\nabla} \wedge \vec{U}, C$ is the pollutant concentration and $\vec{g}$ is the acceleration of gravity. $\mathfrak{R e}, S c, F r$ Respectively represent the Reynolds number, Schmidt and Froude.

\subsection{Application to the problem of a diffuser on the bottom}

On the bottom of a rectangular channel (length $=6 \mathrm{~m}$, depth $\mathrm{L}=4 \mathrm{~m}$ ) was placed a diffuser with $1 \mathrm{~m}$ of diameter. The area of study is limited by two lateral boundaries, a flat free surface and a raincoat horizontal bottom. (Figure 1). The equations to be solved are the equations 1-4. The chosen dimensionless numbers are $\mathrm{F}_{\mathrm{r}}=18, S_{c}=1.5$. The Reynolds number is a parameter of the study. In applying the decomposition method of the field, $\Omega$ is partitioned into two sub areas (Figure 2). In the subdomain $\Omega 1$, we choose to solve the equations $1-4$ by a finite difference method. In $\Omega 2$, we solve the problem by particle method. This choice is justified by the aspect of the flow. In fact, in this subdomain, the aspect of high recirculation speed dominates and the finite difference method is less suited to deal with this aspect of the movement. The particle method is a Lagrangian method adapted to process the convection problems in a turbulent regime (HUBERSON \& CHOQUIN, 1988). The coupling is made by a particle mesh method. 


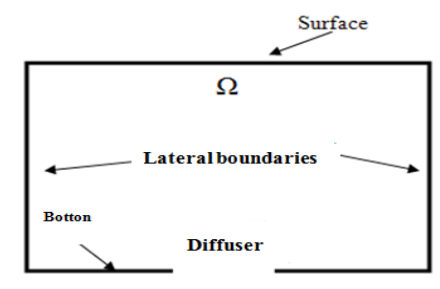

Figure 1. Field of study $\Omega$.

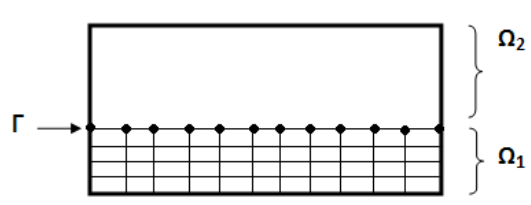

Figure 2. Subdivision of the $\Omega$ domain.

We present respectively the results in the sub area 1 (Figure 3a), at the interface when the fluid starts to leave the field $\Omega 1$ by a weak coupling (Figure 3b) and in the sub domain 2 (Figure 3c) for a Reynolds number equal to 1000. The axes of the units are dimensionless and reported to the diameter of the jet.

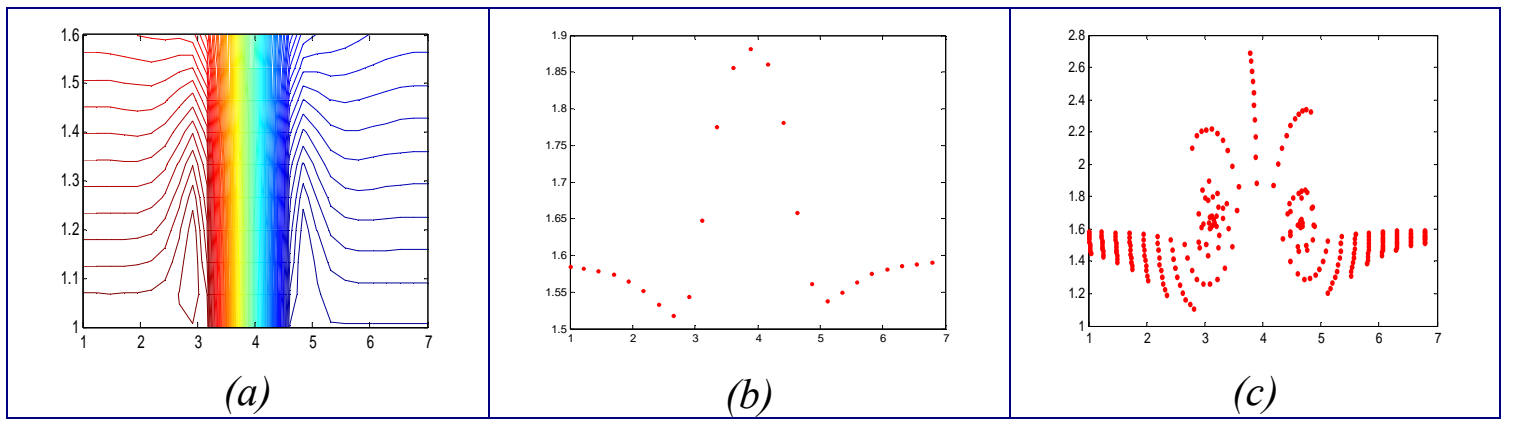

Figure 3. (a) Iso-concentrations in subdomain $\Omega 1$; (b) Position of particle;

(c) Illustration of the method of projection.

We conclude that recoveries of information from the subdomain 1 to the subdomain 2 are made by on a regular and continuous basis. This observation is justified in Figure 4 where the velocity field is represented in the whole area. The intensity of the velocity fields is dimensionless.

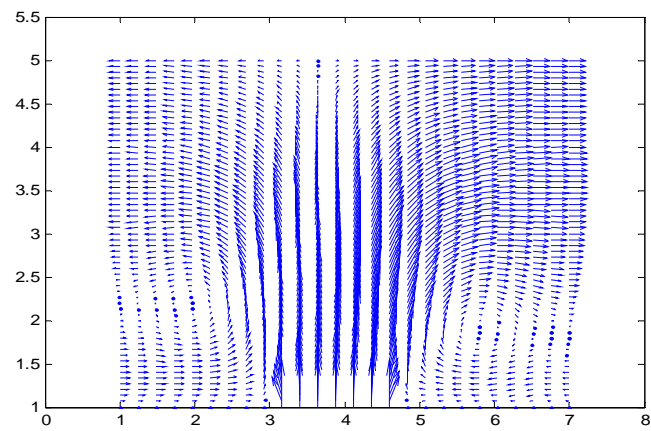

Figure 4. Field of the velocity in the domain of study. 


\subsection{Application to the selective extraction}

This field of study is rectangular, filled with water, $4 \mathrm{~m}$ width and $6 \mathrm{~m}$ length. A jet is taxed upstream and a draw in the downstream part. The geometrical configuration is shown in Figure 5. The objective is to analyze the impact of this mechanism on the recirculation of water in order to eliminate the eutrophication.
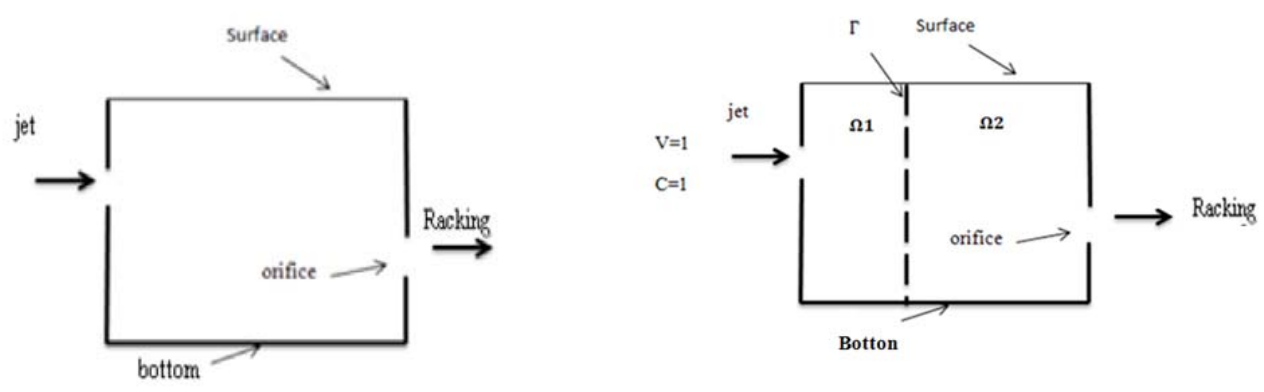

Figure 5. Field of study $\Omega$.

We present in Figure 6 the current lines for a Reynolds number $\Re e=1000$. The recovery of data calculated by the finite difference method is made by regular way from domain 1 to domain 2, when the calculation is done by the particle method. This connection is shown in Figure 7.

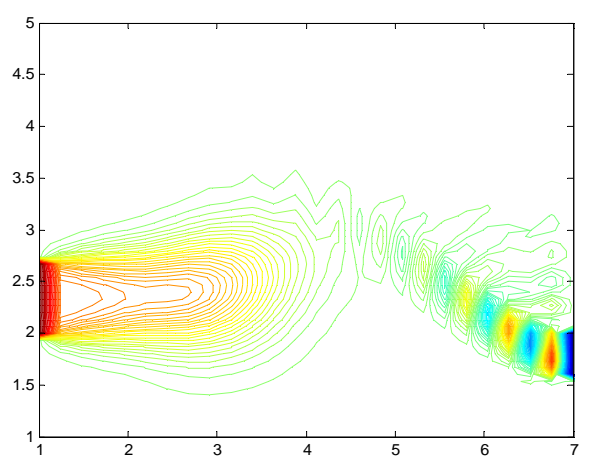

Figure 6. Raking at $t=10 \mathrm{~s}$.

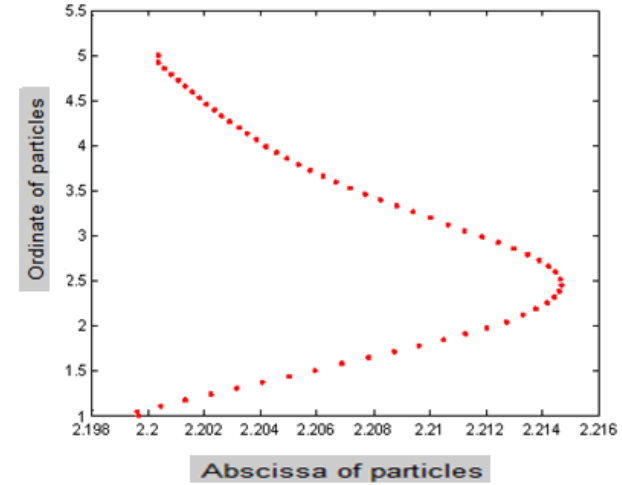

Figure 7. Position of the particle.

\section{Conclusion}

The method of decomposition domain we proposed can analyze and simulate problems for large Reynolds numbers. It is well suited to the problems of confined flows and external flows in the presence of walls. It was applied to two problems governed by two different physical processes: the first is governed by gravity and the second is governed by buoyancy. The extension of this method to various kinds of problems is one of our 
prospects. The main advantage is to perform nonlinear resolutions in large geometrical areas.

\section{References}

ADNAOUI K., TOUNSI N., CHAGDALI M., MORDANE S. (2014). The method of decomposition domain for the digital modelling of a jet. American Journal of Applied Mathematics, Vol. 2(2), pp 64-68. http://dx.doi.org/10.11648/j.ajam.20140202.14

HUBERSON S., CHOQUIN P. (1988). Particles simulations of viscous flow. Computers and Fluids, $\mathrm{n}^{\circ}$ 2, pp 397-410.

MANGOUB G. (2007). Méthode de sous domaine et de décomposition de champs de vitesse: Application aux écoulements à surface libre. Thèse de Doctorat d'État à l’université Hassan II Mohammedia, Faculté Ben M’Sik. 